\title{
DENSE MOLECULAR GAS IN NEARBY GALAXIES
}

\author{
Y. GAO \\ Dept. of Astronomy, Univ. of Illinois and \\ Astronomy Program, SUNY at Stony Brook \\ AND \\ P.M. SOLOMON \\ Astronomy Program, SUNY at Stony Brook
}

\section{Introduction}

HCN, one of the most abundant high dipole-moment molecules (traces molecular gas at densities $\mathrm{n}_{\mathrm{H}_{2}} \gtrsim 3 \times 10^{4} \mathrm{~cm}^{-3}$ whereas $\mathrm{CO}$ traces at $\sim 500$ $\mathrm{cm}^{-3}$ ), has only been detected in $\sim 25$ galaxies, primarily towards the galactic nuclei (Solomon et al. 1992; Nguyen-Q-Rieu et al. 1992; Helfer \& Blitz 1993; Aalto et al. 1995). Dense molecular gas properties in "normal" galactic disks, as compared to the centers of "normal" spirals, starbursts, and luminous IR galaxies are not clear. As part of a large HCN survey in 60 galaxies (Gao 1996; Gao \& Solomon 1997), we present here HCN observations in central regions of a few nearby galaxies. $\mathrm{HCN}$ was mapped at least along the major axes in the inner disks to determine the total HCN luminosity, the distribution with radius of $\mathrm{HCN}$ emission, and of the surface brightness ratio of $\mathrm{SBR} \equiv I_{\mathrm{HCN}} / I_{\mathrm{CO}}$.

\section{Extent and Distribution of Dense Molecular Gas}

There are almost no HCN observations beyond the nuclear $\sim 1 \mathrm{kpc}$ regions in galaxies. We have mapped $\mathrm{HCN}$ along the major axis in $\sim 10$ nearby galaxies. An example is shown in Fig. 1 for M82. Apparently, the dense molecular gas is not confined to the inner $\sim 1 \mathrm{kpc}$ nuclear region, although the highest concentrations of dense molecular gas are in the center. A significant fraction of dense molecular gas is distributed in the inner disks of galaxies outside the nuclear or inner ring starburst regions, and 
can be detected to radii as large as a few kpc (e.g., in NGC 891), perhaps to diameters of $\sim D_{25} / 4$.

The radial distribution of HCN emission and the SBR in NGC 253 obtained from both the IRAM $30 \mathrm{~m}$ and NRAO $12 \mathrm{~m}$ are presented in Fig. 2 . The HCN distribution in the innermost part of disks of galaxies seems to follow approximately an exponentially decreasing law. However, it seems to be more difficult to describe the SBR as a function of radius.

\section{Starburst Indicator $\mathrm{SBR} \equiv I_{\mathrm{HCN}} / I_{\mathrm{CO}}$}

The SBR in NGC 253 reveals a nuclear starburst core of highest SBR $\sim 0.1$ with a linear scale $\lesssim 0.5 \mathrm{kpc}$. At larger radius, $\mathrm{R} \gtrsim 0.3 \mathrm{kpc}$, the fraction of dense molecular gas becomes smaller and the SBR drops to the global value for normal spirals like NGC $891(\sim 0.025)$, which shows slowly declining but almost constant SBR across the inner disk (on a linear scale of $\sim 5 \mathrm{kpc}$ ). In galaxies like M82 and NGC 6946, a rather constant SBR $\sim 0.04-0.05$ (moderate starburst) across the innermost a few kpc region is observed, whereas normal galaxies and even the outer part of the inner disks of the starburst galaxies (e.g., NGC 253) have SBR about $0.02-0.03$ (non-starburst, normal).

Ultraluminous IR galaxies have the highest $\mathrm{HCN}$-to-CO luminosity ratio, $L_{\mathrm{HCN}} / L_{\mathrm{CO}} \sim 0.1-0.3$ (Solomon et al. 1992; Gao \& Solomon 1997), similar to the SBR in the starburst cores, whereas "normal" spirals have a ratio $L_{\mathrm{HCN}} / L_{\mathrm{CO}} \sim 0.01-0.05$, same as the SBR in non-starburst galaxies or the disks outside the starburst regions.

Therefore, the SBR seems to be an indicator of the starburst and the radial distribution of SBR as a function of galactic radius may suggest the starburst size scales in galaxies.

\section{References}

Aalto, S., et al. 1995, A\&A, 300, 369.

Gao, Y. 1996 Ph.D. thesis, SUNY at Stony Brook.

Gao, Y. \& Solomon, P.M. 1997 ApJ, submitted.

Helfer, T.T., \& Blitz, L. 1993, ApJ, 419, 86.

Nguyen-Q-Rieu, et al. 1992, ApJ, 399, 521.

Solomon, P.M., Downes, D. \& Radford, S.J.E. 1992, ApJ, 387, L55. 

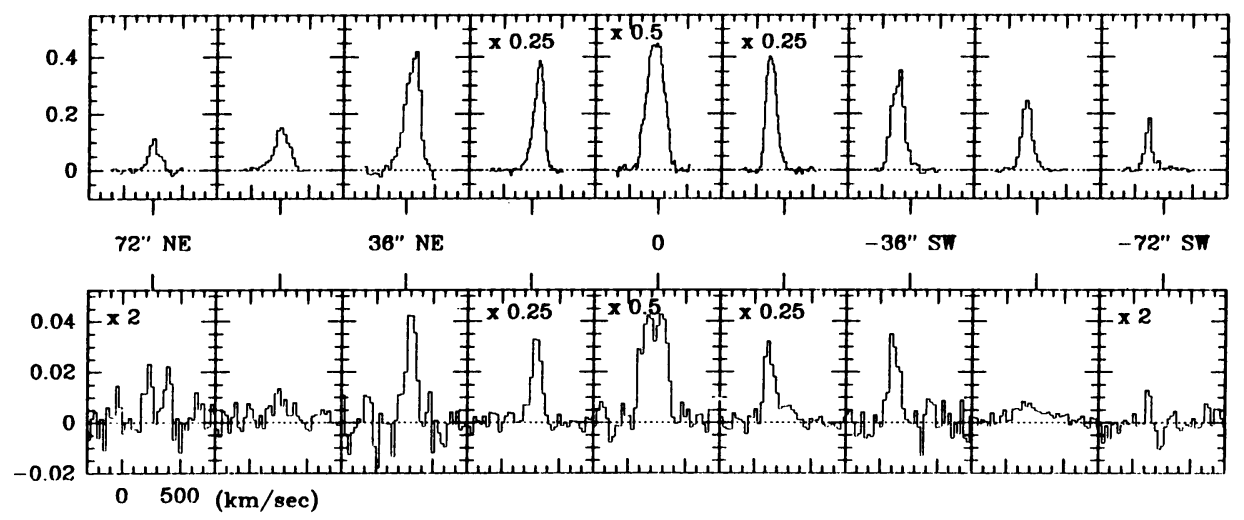

Figure 1. HCN spectra (bottom) mapped along the major axis in M82 as compared with the $\mathrm{CO}(2-1)$ spectra (top) obtained simultaneously from the IRAM $30 \mathrm{~m}$ telescope. The vertical scale is the antenna brightness temperature $T_{A}^{*}(\mathrm{~K})$.
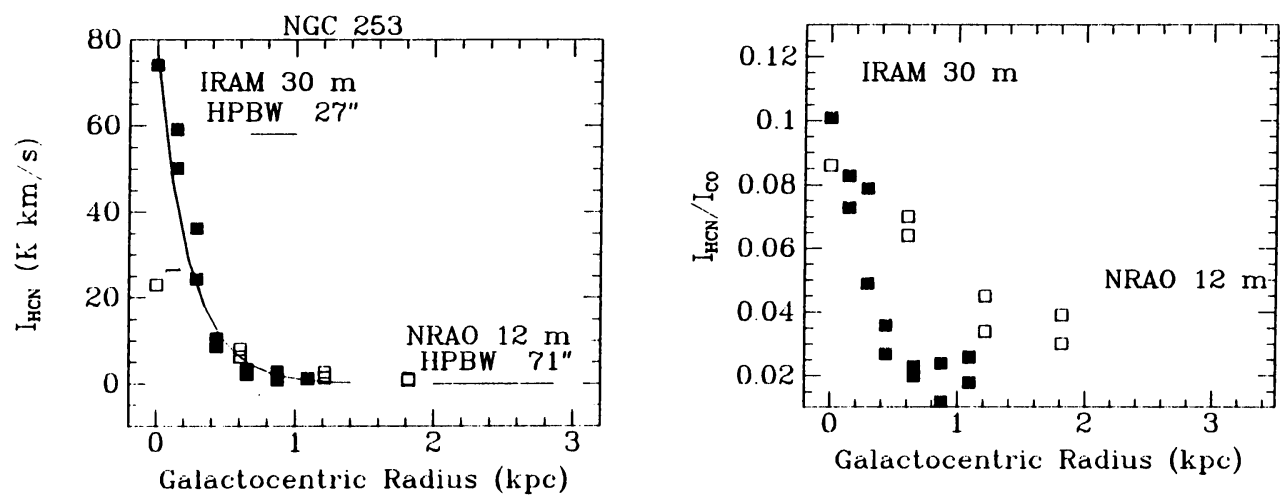

Figure 2. HCN emission (the integrated line intensity) and the surface brightness ratio $\mathrm{SBR} \equiv I_{\mathrm{HCN}} / I_{\mathrm{CO}}$ as a function of the galactic radius in NGC 253 (filled squares are the IRAM $30 \mathrm{~m}$ data and the open squares are the NRAO $12 \mathrm{~m}$ observations). The line is an exponential fit. 